\title{
An all-sky search of EXPLORER data
}

\author{
P Astone $^{1}$, D Babusci $^{2}$, M Bassan $^{3}, \mathbf{K}$ M Borkowski ${ }^{4}$, L Brocco $^{5}$,

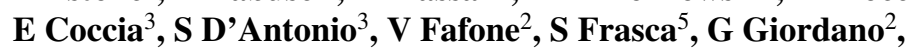 \\ P Jaranowski ${ }^{6}$, A Królak ${ }^{7,10}$, A Marini ${ }^{2}$, Y Minenkov ${ }^{3}$, I Modena ${ }^{3}$, \\ G Modestino $^{2}$, A Moleti ${ }^{3}$, A Pai ${ }^{1}$, G V Pallottino ${ }^{5}$, C Palomba ${ }^{1}$, M Pietka ${ }^{6}$,

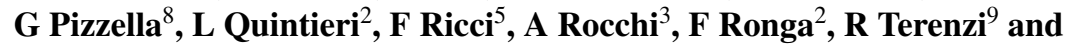 \\ M Visco ${ }^{9}$ \\ ${ }^{1}$ Istituto Nazionale di Fisica Nucleare INFN, Rome, Italy \\ 2 Istituto Nazionale di Fisica Nucleare INFN, Frascati, Italy \\ ${ }^{3}$ University of Rome 'Tor Vergata' and INFN, Rome II, Italy \\ ${ }^{4}$ Center for Astronomy, Nicolaus Copernicus University, Toruń, Poland \\ ${ }^{5}$ University of Rome 'La Sapienza' and INFN, Rome, Italy \\ ${ }^{6}$ Institute of Theoretical Physics, University of Białystok, Białystok, Poland \\ ${ }^{7}$ Albert Einstein Institute, Golm, Germany \\ ${ }^{8}$ University of Rome 'Tor Vergata' and INFN, Frascati, Italy \\ ${ }^{9}$ IFSI-CNR and INFN, Rome \\ E-mail: krolan@aei.mpg.de
}

Received 15 April 2005, in final form 28 June 2005

Published 6 September 2005

Online at stacks.iop.org/CQG/22/S1243

\begin{abstract}
We have analysed three data sets, each two days long, of the EXPLORER resonant bar detector. We have searched for continuous gravitational-wave signals from spinning neutron stars. Our data analysis technique was based on the maximum likelihood detection method. We briefly describe the theoretical methods that we used in our search and we present results of the search. The main outcome of our analysis is an upper limit of $1 \times 10^{-22}$ for the dimensionless amplitude of a continuous gravitational-wave signal. The upper limit is for any source location in the sky, any polarization of the wave and for signals of frequency from $921.00 \mathrm{~Hz}$ to $921.76 \mathrm{~Hz}$ and with spin down from $-2.36 \times 10^{-8} \mathrm{~Hz} \mathrm{~s}^{-1}$ to $+2.36 \times 10^{-8} \mathrm{~Hz} \mathrm{~s}^{-1}$.
\end{abstract}

PACS numbers: 95.55.Ym, 04.80.Nn, 95.75.Pq, 97.60.Gb

(Some figures in this article are in colour only in the electronic version)

${ }^{10}$ On leave of absence from Institute of Mathematics Polish Academy of Sciences, Warsaw, Poland. 


\section{Introduction}

When we observe a continuous gravitational-wave signal by a ground-based detector for a sufficiently long time its phase and amplitude are modulated due to the motion of the detector with respect to the solar system barycentre. Therefore, detecting such a signal and estimating its parameters enables determination of the position of the source of the wave. Further, in the data from a single detector gravitational waves from all sky locations are registered. This enables an all-sky search for continuous gravitational waves with a single detector. The difficulty in the search for gravitational-wave signals is that they are very weak and they are deeply buried in the noise of the detector. Consequently, the detection of these signals and interpretation of data analysis results is a delicate task. In this paper, we present results of an all-sky search for continuous sources of gravitational radiation. A prime example of such a source is a spinning neutron star. A signal from such a source has definite characteristics that make it suitable for application of the optimal detection techniques based on matched filtering. Moreover, such signals are stable as a result of the stability of the rotation of the neutron star and they will be present in the data for time periods much longer than the observational interval. This enables a reliable verification of the potential candidates by repeating the observations both by the same detector and by different detectors.

In our all-sky search, we have used the data of the EXPLORER resonant bar detector [1]. A directed search of the galactic centre with the EXPLORER detector has already been carried out and an upper limit for the amplitude of the gravitational waves has been established [2].

This paper is organized as follows. In section 2 we summarize the theoretical tools that we use in our analysis, in section 3 we present results of our all-sky search, and in section 4 we derive the main result of our analysis which is an upper limit for the dimensionless amplitude of continuous gravitational waves from spinning neutron stars. This is a revised upper limit with respect to one we previously reported for a shorter period [7].

The data analysis was performed by a team consisting of Pia Astone, Kaz Borkowski, Piotr Jaranowski, Andrzej Królak and Maciej Pietka and was carried out on the basis of a Memorandum of Understanding between the ROG Collaboration and Institute of Mathematics of Polish Academy of Sciences. More details about the search can be found in [9].

\section{Data analysis methods}

In this section, we give a summary of data analysis techniques that we used in the search. More details are given in [3-7].

\subsection{Response of a bar detector to a continuous gravitational-wave signal}

The dimensionless noise-free response function $h$ of a resonant bar gravitational-wave detector to a weak plane gravitational wave in the long wavelength approximation (i.e., when the size of the detector is much smaller than the reduced wavelength $\lambda /(2 \pi)$ of the wave) can be written in the following form:

$$
h(t)=\sum_{i=1}^{4} A_{i} h_{i}(t)
$$

where $A_{i}$ are four constant amplitudes that depend on amplitude $h_{o}$ and phase $\phi_{o}$ of the wave and two polarization angles $\iota$ and $\psi$. The time-dependent functions $h_{i}$ have the form

$$
\begin{aligned}
& h_{1}(t)=a(t) \cos \Phi(t), \quad h_{2}(t)=b(t) \cos \Phi(t), \\
& h_{3}(t)=a(t) \sin \Phi(t), \quad h_{4}(t)=b(t) \sin \Phi(t) .
\end{aligned}
$$


The functions $a$ and $b$ are amplitude modulation functions (see equations (3) of [7]), and $\Phi$ is the phase modulation (see equation (6) of [7]). The amplitude modulations $a$ and $b$ depend on the right ascension $\alpha$ and the declination $\delta$ of the source and they vary with a period of one sidereal day. The phase $\Phi$ depends on the frequency $\omega_{0}$, the $s$ spin-down parameters $\omega_{k}(k=1, \ldots, s)$ and on the angles $\alpha, \delta$. We call the parameters $\omega_{0}, \omega_{k}, \alpha, \delta$ the intrinsic parameters and the remaining ones the extrinsic parameters. As we shall see in the following section we only need to search for signals over the intrinsic parameter space. The whole signal $h$ depends on $s+7$ unknown parameters: $h_{o}, \phi_{o}, \iota, \psi, \alpha, \delta, \omega_{0}, \omega_{k}$. The response also depends on the position of the detector with respect to the solar system barycentre (SSB). This position can be determined with a great accuracy using JPL Planetary and Lunar Ephemerides DE405/LE405.

\subsection{Optimal data analysis method}

Assuming that the noise in the detector is an additive, stationary, Gaussian and zero-mean random process, we can derive the optimum matched filter for the signal given in section 2.1. Under a simplifying assumption that the observation time $T_{o}$ is an integer multiple of 1 sidereal day and assuming that over the bandwidth of the signal $h$ the spectral density $S_{h}(f)$ of the detector's noise is constant and equal to $S_{h}\left(f_{0}\right)$, where $f_{0}$ is the frequency of the signal measured at the SSB at the beginning time of the analysis, the application of the matched filter reduces to evaluating the $\mathcal{F}$-statistic given by

$$
\mathcal{F}=\frac{2}{S_{h}\left(f_{0}\right) T_{o}}\left(\frac{\left|F_{a}\right|^{2}}{\left\langle a^{2}\right\rangle}+\frac{\left|F_{b}\right|^{2}}{\left\langle b^{2}\right\rangle}\right),
$$

where

$$
\begin{aligned}
& F_{a}:=\int_{0}^{T_{o}} x(t) a(t) \exp [-\mathrm{i} \Phi(t)] \mathrm{d} t, \\
& F_{b}:=\int_{0}^{T_{o}} x(t) b(t) \exp [-\mathrm{i} \Phi(t)] \mathrm{d} t .
\end{aligned}
$$

In equation (4) $x(t)$ is the $h$-reconstructed data and $\langle\cdot\rangle$ denotes time averaging over the observational interval $\left[0, T_{o}\right]$.

The $\mathcal{F}$-statistic given above depends only on the intrinsic parameters whereas the estimators of the four amplitudes $A_{i}$ are given in an explicit analytic form:

$$
\widehat{A}_{1}=2 \frac{\left\langle x h_{1}\right\rangle}{\left\langle a^{2}\right\rangle}, \quad \widehat{A}_{2}=2 \frac{\left\langle x h_{2}\right\rangle}{\left\langle b^{2}\right\rangle}, \quad \widehat{A}_{3}=2 \frac{\left\langle x h_{3}\right\rangle}{\left\langle a^{2}\right\rangle}, \quad \widehat{A}_{4}=2 \frac{\left\langle x h_{4}\right\rangle}{\left\langle b^{2}\right\rangle} .
$$

The estimators of the signal parameters are obtained in two steps. Firstly, the estimators of the intrinsic parameters (frequency, spin-down parameters, and the angles $\alpha$ and $\delta$ ) are obtained by maximizing the functional $\mathcal{F}$ with respect to these parameters. Secondly, the estimators of the amplitudes $A_{i}$ are calculated from the analytic formulae (5) with the correlations $\left\langle x h_{i}\right\rangle$ evaluated for the values of the parameters obtained in the first step.

\subsection{An approximate model}

In order to calculate the $\mathcal{F}$-statistic efficiently, we introduced an approximation to the phase $\Phi(t)$ of the signal consisting of expansion of the motion of the Earth around the Sun in a Taylor series. We find that for a two-day long data set the following approximation is satisfactory:

$$
\Psi_{s}(t)=p+p_{0} t+p_{1} t^{2}+A \cos \left(\Omega_{r} t\right)+B \sin \left(\Omega_{r} t\right),
$$


where $\Omega_{r}$ is the rotational angular velocity of the Earth. The characteristic feature of the above approximation is that the phase is a linear function of the parameters of the signal. The parameters $A$ and $B$ are related to the right ascension $\alpha$ and the declination $\delta$ of the gravitational-wave source through the equations

$$
A=\frac{\omega_{0} r}{c} \cos \delta \cos \left(\alpha-\phi_{r}\right), \quad B=\frac{\omega_{0} r}{c} \cos \delta \sin \left(\alpha-\phi_{r}\right),
$$

where $\omega_{0}$ is the angular frequency of the gravitational-wave signal and $r$ is the equatorial component of the detector's radius vector with respect to the centre of the Earth. The parameters $p, p_{0}$ and $p_{1}$ contain contributions both from the intrinsic evolution of the gravitational-wave source and the modulation of the signal due to the motion of the Earth around the Sun.

\subsection{Search strategy}

With the above approximation, the integrals given by equations (4) that we needed to compute in order to evaluate $\mathcal{F}$ are Fourier transforms and they can be efficiently calculated using the FFT algorithm. Thus, the evaluation of $\mathcal{F}$ consists of correlation of the data with two linear filters depending on parameters $p_{1}, A, B$ followed by FFTs. In [6], we have verified that for the case of our search the linear approximation to the phase does not produce a loss in signal-to-noise ratio of more than 5\%. The linear approximation is the better the shorter the observation time, the narrower the bandwidth and the higher the frequency of the signal.

In order to identify potential gravitational-wave signals, we apply a two-step procedure consisting of a coarse search followed by a fine search. The coarse search consists of evaluation of $\mathcal{F}$ on a discrete grid in the intrinsic parameter space and comparison of the obtained values of $\mathcal{F}$ with a predefined threshold $\mathcal{F}_{o}$. The grid is constructed in such a way that the loss of the signal-to-noise ratio is minimized. The parameters of the nodes of the grid for which the threshold is crossed are registered. These events are called triggers of our search. The threshold is calculated from a chosen false alarm probability which is defined as the probability that $\mathcal{F}$ crosses the threshold when no signal is present and the data are only noise. The fine search consists of finding a local maximum of $\mathcal{F}$ for each trigger using a numerical implementation of the Nelder-Mead algorithm [10], where coordinates of the starting point of the maximization procedure are the parameter values of the coarse search.

\subsection{False alarm probability, detection probability, grid of templates}

To calculate the false alarm probability as a function of the threshold and to construct a grid in the parameter space we introduce yet another approximation of our signal. Namely, we use a signal with a constant amplitude and the phase given by equation (6). In paper [4], we have shown that the Fisher matrix for the exact model with amplitude modulations given by equation (1) can be accurately reproduced by the Fisher matrix of a constant amplitude model. As the calculations of the false alarm probability and construction of a grid in the parameter space are based on the Fisher matrix, we expect that the constant amplitude model is a good approximation for the purpose of the above calculations. We stress that in the search of real data we used the full model with amplitude modulations.

False alarm probability $P_{F}^{T}\left(\mathcal{F}_{o}\right)$ is the probability that the $\mathcal{F}$-statistic crosses a threshold $\mathcal{F}_{o}$ and is given by

$$
P_{F}^{T}\left(\mathcal{F}_{o}\right)=1-\left[1-P_{F}\left(\mathcal{F}_{o}\right)\right]^{N_{c}},
$$

where $N_{c}$ is the number of cells in the parameter space, i.e., the number of statistically independent realizations of the $\mathcal{F}$-statistic when the data are only noise and $P_{F}$ is false alarm 
probability for a single cell. If the detector noise is Gaussian, $2 \mathcal{F}$ has $\chi^{2}$ distribution with four degrees of freedom and $P_{F}$ is given by

$$
P_{F}\left(\mathcal{F}_{o}\right)=\left(1+\mathcal{F}_{o}\right) \exp \left(-\mathcal{F}_{o}\right)
$$

Probability of detection $P_{D}\left(d, \mathcal{F}_{o}\right)$ is the probability that a signal with signal-to-noise ratio $d$ crosses the threshold $\mathcal{F}_{o}$ and is given by

$$
P_{D}\left(d, \mathcal{F}_{o}\right):=\int_{\mathcal{F}_{o}}^{\infty} p_{1}(d, \mathcal{F}) \mathrm{d} \mathcal{F}
$$

where (here $I_{1}$ is the modified Bessel function of the first kind and order one)

$$
p_{1}(d, \mathcal{F})=\frac{\sqrt{2 \mathcal{F}}}{d} I_{1}(d \sqrt{2 \mathcal{F}}) \exp \left(-\mathcal{F}-\frac{1}{2} d^{2}\right) .
$$

We choose the grid of templates in such a way that the correlation $C$ between any potential signal present in the data and the nearest point of the grid never falls below a certain value. In the case of the approximate model of the signal that we use, the grid is uniform and consists of regular polygons in the space parametrized by $p_{0}, p_{1}, A, B$. The construction of the grid is described in detail in section VIIA of [2]. For the grid that we used in the search of the EXPLORER data the correlation function $C$ for any signal present in the data was greater than 0.77 .

\section{An all-sky search of EXPLORER data}

We have implemented the theoretical tools presented in section 2 and we have performed an all-sky search for continuous sources of gravitational waves in the data of the resonant bar detector EXPLORER ${ }^{11}$ [1]. The detector has collected many years of data with a high duty cycle (e.g., in 1991 the duty cycle was 75\%). The EXPLORER detector was, in the year 1991, most sensitive for two narrow bandwidths (called minus and plus modes) of about $1 \mathrm{~Hz}$ wide at two frequencies around $1 \mathrm{kHz} .{ }^{12}$ To make the search computationally manageable, we analysed two days of data in the narrow band where the detector had the best sensitivity. To narrow band the data, we use the FFT database in which EXPLORER data are stored and extract the data by doing inverse FFT of the Fourier data for a bandwidth of our choice. The procedure of extraction of a narrow band sequence from the FFT database is described in detail in [2], appendix C. By narrowing the bandwidth of the search we can shorten the length of the data to be analysed as we need to sample the data at the rate of only twice the bandwidth [8].

For the sake of the FFT algorithm, it is best to keep the length of the data to be a power of 2. Consequently, we have chosen the number of data points to analyse to be $N=2^{18}$. Thus for $T_{o}=2$ days of observation time the bandwidth $\Delta v$ was $\Delta v=N /\left(2 T_{o}\right) \sim 0.76 \mathrm{~Hz}$. We analysed the data around the plus resonance of the system, because in the chosen period it was most sensitive, compared to the minus resonance. The same analysis could be repeated around the minus resonance. As a result, we searched the bandwidth from $921.00 \mathrm{~Hz}$ to $921.76 \mathrm{~Hz}$. We have included one spin-down parameter in the search and we have searched the spin-down range from $-2.36 \times 10^{-8} \mathrm{~Hz} \mathrm{~s}^{-1}$ to $+2.36 \times 10^{-8} \mathrm{~Hz} \mathrm{~s}^{-1}$. We have first searched one two-day stretch of data. The first results of that analysis were reported in [7]. We have then performed two further searches of two-day stretches of data.

\footnotetext{
${ }^{11}$ The EXPLORER detector is operated by the ROG Collaboration of the Italian Istituto Nazionale di Fisica Nucleare (INFN); see http://www.roma1.infn.it/rog/.

${ }^{12}$ In 2000, the EXPLORER detector was upgraded and its high sensitivity was extended to a bandwidth of $10 \mathrm{~Hz}$.
} 


\subsection{Parameter space}

We have used the filters with the phase linear in the parameters given by equation (6). In the filters, we have included the amplitude modulation. The number of cells $N_{c}$ in parameter space, which is the number of independent realizations of the statistic $\mathcal{F}$ when there is no signal in the data, was around $1.6 \times 10^{12}$. Consequently from equation (8), the threshold signal-to-noise ratio for $1 \%$ false alarm probability was equal to 8.3 . In the search that we have performed we have used a lower threshold signal-to-noise ratio of 6.7. The aim of lowering the threshold was to make up for the loss of the signal-to-noise ratio due the discreteness of the grid of templates and due to the use of filters that only approximately matched the true signal. The number of points in the grid over which we had to calculate the statistic $\mathcal{F}$ turned out to be 183064440 . This number involved 63830 positions in the sky and 2868 spin-down values for each sky position.

\subsection{Data selection}

The two-day stretches of data that we have analysed were taken from a larger set of 13 days of data taken by the EXPLORER detector in November 1991. We have first carried out a characterization of the data. We have divided the data into $2^{16}$ points sections corresponding to around $11 \mathrm{~h}$ of data. For each stretch, we have obtained a box-and-whisker plot, estimated spectral density, and performed the Kolmogorov-Smirnov (KS) test.

The box-and-whisker plots display the amount of outliers present in the data. Each box has lines at the lower quartile, median and upper quartile values of the data stretch. The whiskers are lines extending from each end of the box to show the extent of the rest of the data. Outliers are data with values beyond the ends of the whiskers and they are marked by $\mathrm{a}+$ sign. The whisker extends to the most extreme data value within 1.5 interquartile range of the box. Spectral density gives us sensitivity of the detector at a given frequency. The KS test calculates the KS distance between the sample distribution and the Gaussian distribution and tests the null hypothesis that the data come from the Gaussian distribution. The output of the test is the $p$-value which is the probability of observing the KS distance under the null hypothesis. Thus, the higher the $p$-value the more 'Gaussian' the data are.

The results of the characterization of our data are presented in figure 1. From the KS test, we conclude that large parts of our data are approximately Gaussian. We have chosen the three two-day long stretches of data on the basis of conformity of the data to the Gaussian random distribution. The stretches we have chosen are marked by vertical lines in figure 1 . The modified Julian dates of the first samples of the three sets are $48580.7909,48590.3221$, and 48582.7854 , respectively.

In figure 2, we have presented the spectral densities of the two-day stretches of data that we have chosen. We see that the minimum spectral density was close to $10^{-42} \mathrm{~Hz}^{-1}$.

\subsection{Results}

In table 1, we present numbers of triggers in the three searches for threshold corresponding to signal-to-noise ratio of 6.7. We recall that this threshold is lower than the threshold of 8.3 corresponding to false alarm probability of $1 \%$. In figure 3, we have plotted the histograms of the values of the $\mathcal{F}$-statistic for the independent candidates and we have compared them with the theoretical distribution for $\mathcal{F}$ when no signal is present in the data. We see that we obtain a good agreement with the theoretical distribution of $\frac{1}{2} \chi^{2}$ with four degrees of freedom only for the first set of data that we have analysed. The other two data sets show non-Gaussian behaviour which is most prominent for the third set. 

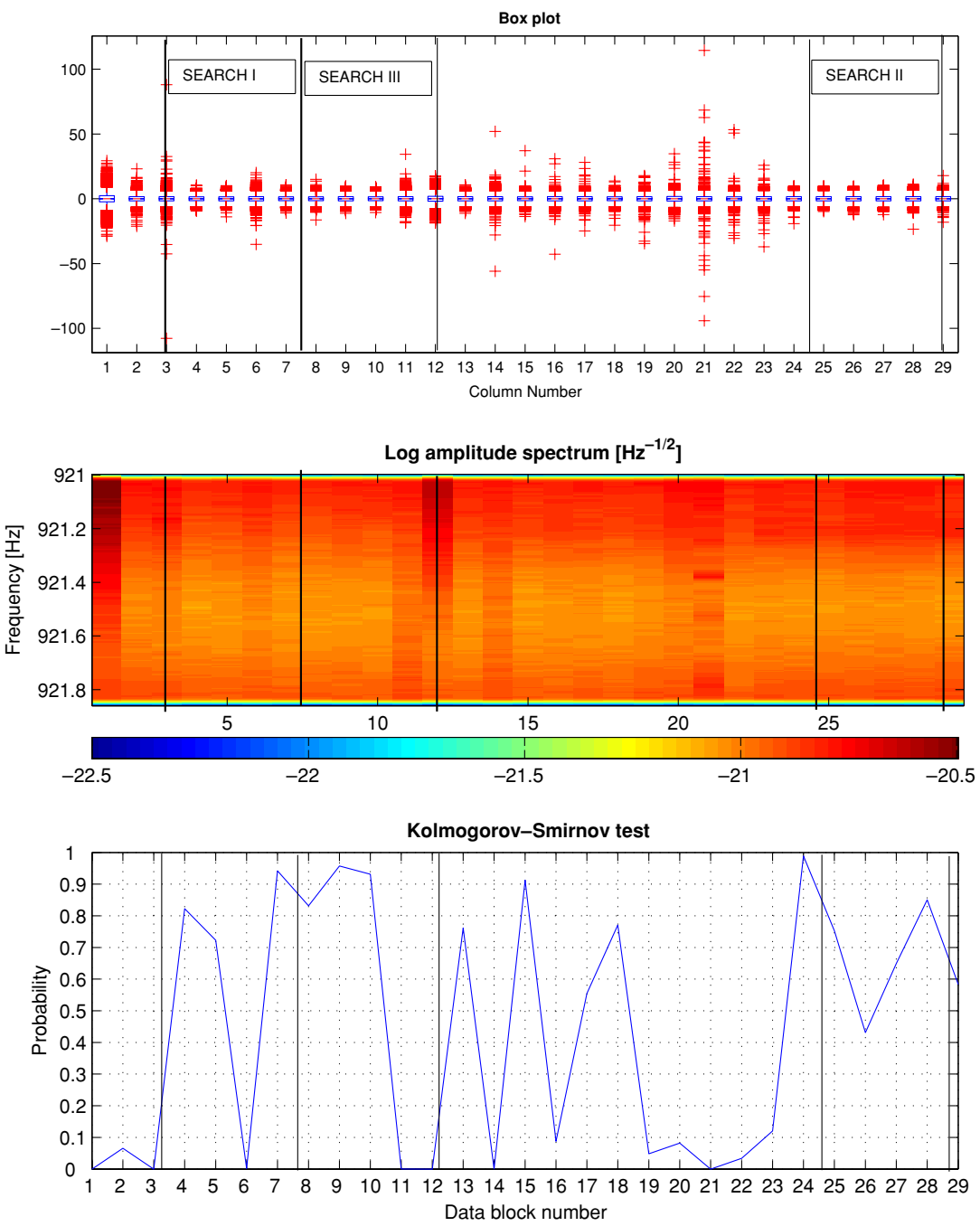

Figure 1. Quality of the EXPLORER data. The $x$-axis gives the number of the 11-h block of data from the 13-day data run. The top panel is the box plots for each block of data. The middle panel is the spectrogram of the data. The spectrogram contains the spectrum of each block of data. The bottom panel gives results of the KS test. The vertical lines indicate the beginning and end of each search that we have performed.

Table 1. Number of triggers for the three searches of EXPLORER data.

\begin{tabular}{lll}
\hline Search & Northern hemisphere & Southern hemisphere \\
\hline I & 15663 & 21601 \\
II & 9883 & 11666 \\
III & 18776 & 23850 \\
\hline
\end{tabular}

The next step of our analysis is the verification of the triggers using filters based on the accurate model of the signal presented in the previous section. The verification procedure consisted of five steps. 


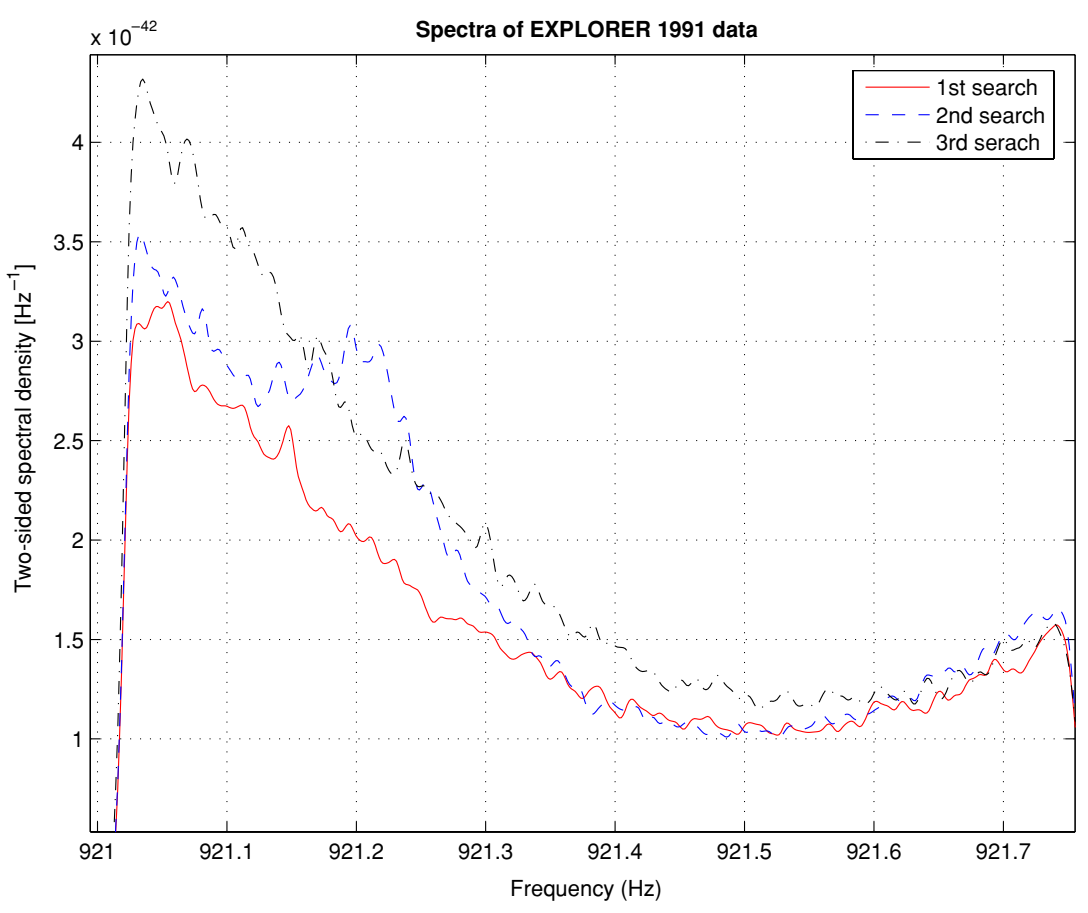

Figure 2. Two-sided spectral density of the three two-day stretches of EXPLORER data that were analysed.

(i) Fine search using the linear filter.

(ii) Fine search with accurate templates that includes precise detector ephemeris.

(iii) Fine search for the signal in another two-day stretch of the available data.

(iv) Fine search of a four-day stretch of data consisting of the original one and the two-day stretch of data following it.

(v) Fine search in the whole data set.

In the first search no event has crossed our $99 \%$ confidence threshold of 8.285 . In the second search there was one event crossing; however, the event has not crossed the threshold of 6.7 in a different data set and in the four-day stretch the event has not even crossed the threshold of 6.7. For the third search there were 76 threshold crossings. There were no corresponding crossings of the threshold of 6.7 in a different data set and for four days of observation time highest increase in signal-to-noise ratio was 5\%. Typically, the signal-tonoise ratio for four days has decreased by around $15 \%$. Consequently, we can attribute the 8.285 threshold crossings to non-Gaussian behaviour of the noise as the $99 \%$ confidence of this threshold was calculated assuming the data were Gaussian.

The results of the verification procedure for one of the triggers are presented in figure 4 . A trigger of signal-to-noise ratio 7.4, crossing of a low threshold of 6.7 , occurred. In astrophysical parametrization, an event of signal-to-noise ratio of 7.3, crossing our threshold, also occurred. There was an event in a different stretch of data of somewhat lower signal-tonoise ratio of 6.7. However, for four days of data signal-to-noise ratio decreased with respect to the signal-to-noise ratio for two days. Consequently, we do not consider this trigger as a gravitational-wave signal candidate. 
Distribution of trigger events
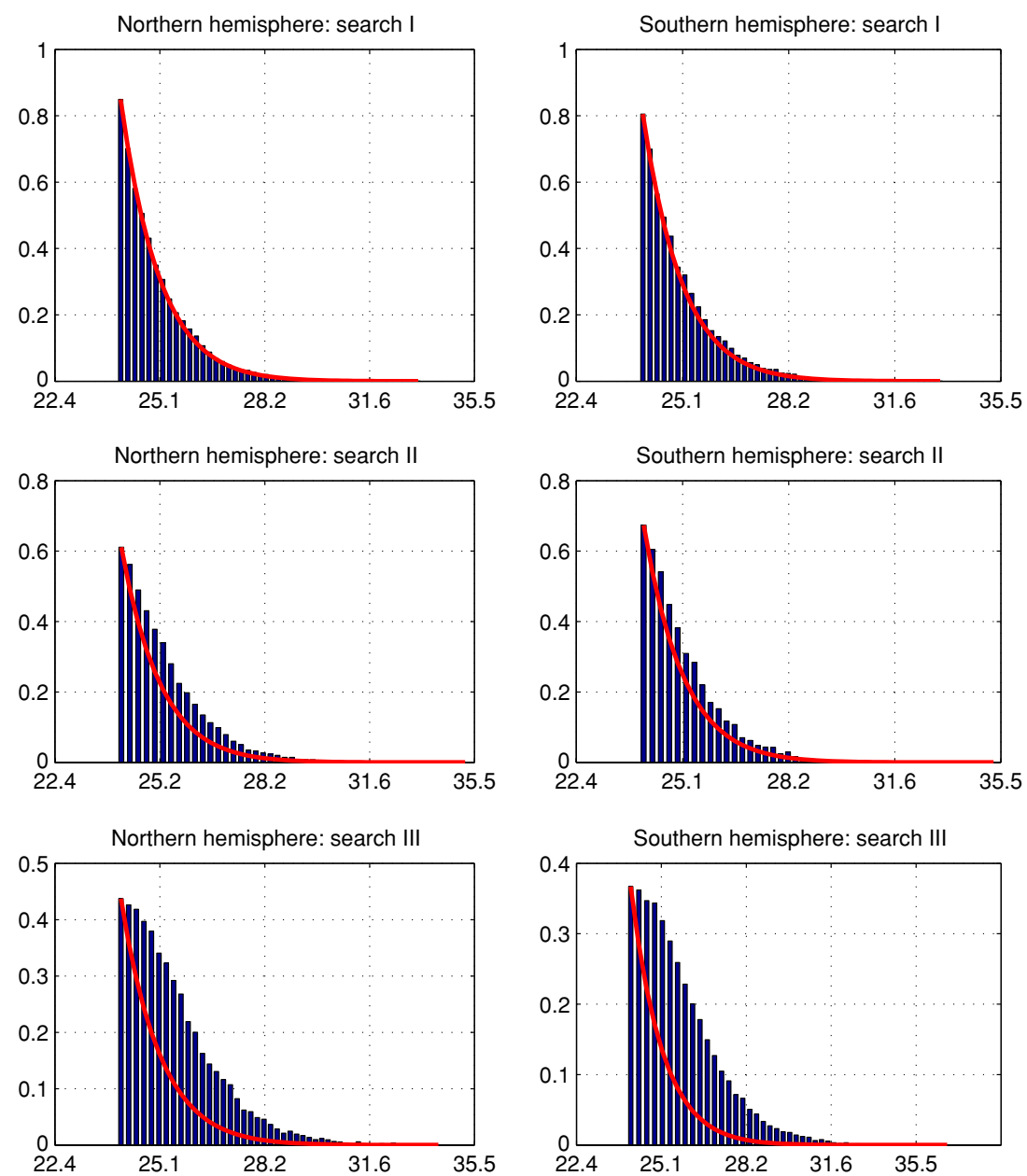

Figure 3. Histograms of the values of the $\mathcal{F}$-statistic of triggers for the three searches of EXPLORER data. The solid line corresponds to the theoretical $\frac{1}{2} \chi^{2}$ distribution with four degrees of freedom. The $x$-axis gives values of the $\mathcal{F}$-statistic.

In order to make sure that we do not miss any real signals among our trigger events we have carried out the verification procedure of all the triggers in the whole 13-day long data set available for us. If any of the triggers were a true gravitational-wave signal, we should have obtained very strong signals of SNR equal to 17 or more. The largest signal-to-noise ratio we obtained for all three searches was 7.7.

In table 2, we have given values of maximum signal-to-noise ratio for all events at various stages of search and verification procedure and for all the three searches that we have performed.

\section{Upper limit}

As we do not have a detection of a gravitational-wave signal, we can make a statement about the upper bound for the gravitational-wave amplitude. We calculate our upper limit assuming 

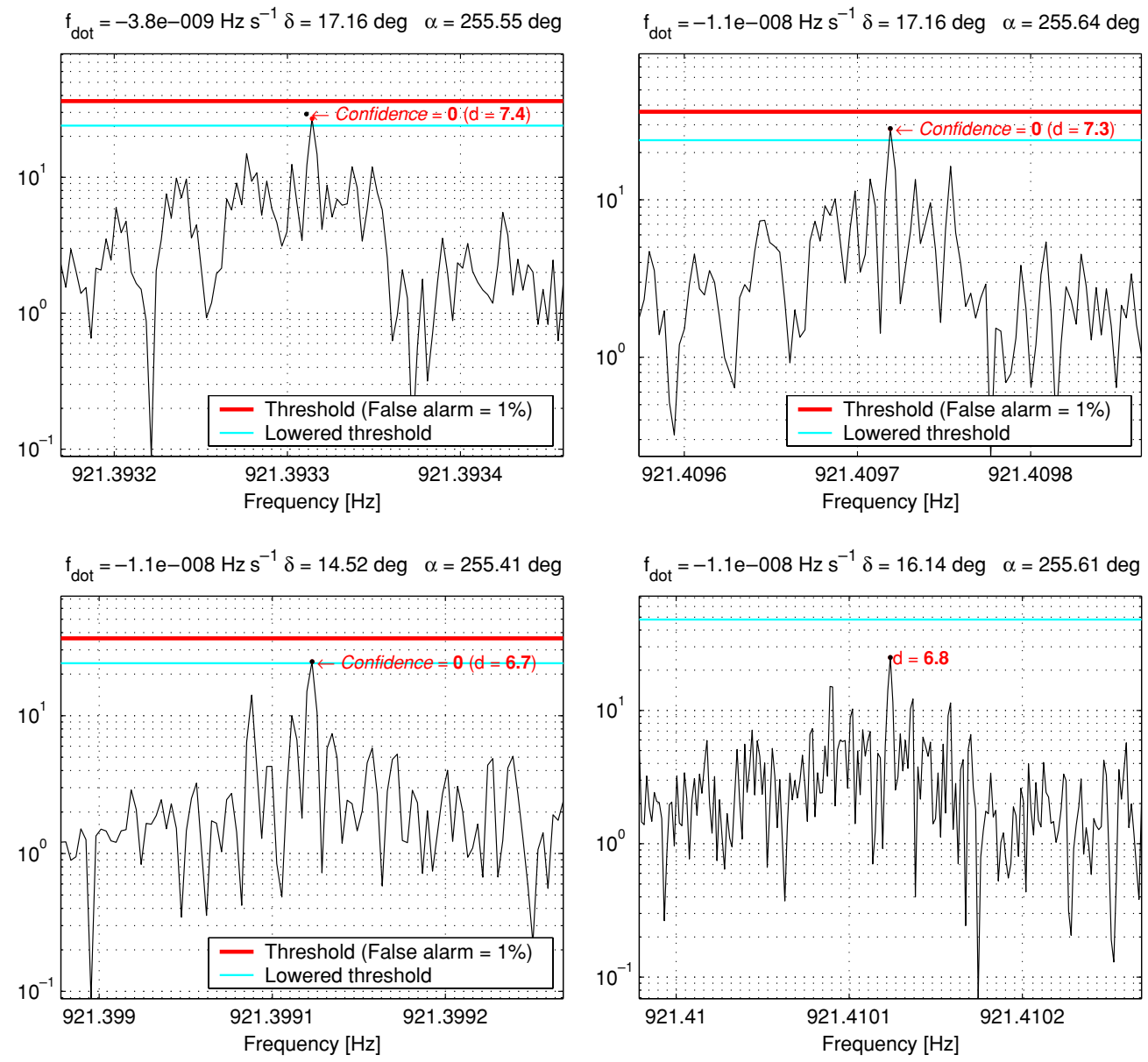

Figure 4. An example of verification procedure of a trigger signal. The four panels are plots of $\mathcal{F}$-statistic as a function of frequency. Signal-to-noise ratios and confidence levels corresponding to maxima of the $\mathcal{F}$-statistic are given. The threshold corresponding to $1 \%$ false alarm probability is drawn by a horizontal thick line. The thin horizontal line denotes lowered threshold equivalent to signal-to-noise ratio of 6.7. For four days verification procedure, the thin vertical line corresponds to signal-to-noise ratio equal to $\sqrt{2} \times 6.7$. The left top panel is for approximate linear parametrization of the signal, the right top panel is for astrophysical parameters. Note the difference in the frequency of the trigger for the two panels. The frequency in linear parametrization contains the contribution from the motion of the Earth around the Sun. The left bottom panel is the result of verification of the trigger in a different data set. The right bottom panel is verification in a twice as long data set. The trigger is not considered as a gravitational-wave candidate because it is not a significant signal in the two-day search and its signal-to-noise ratio does not increase when we increase the observation time by a factor of two. In fact the signal-to-noise ratio decreased by $5 \%$ instead of increasing (theoretically by a factor of $\sqrt{2}$ ).

Gaussian distribution of the detector's noise. We take a threshold value $\mathcal{F}_{o}$ of the $\mathcal{F}$-statistic corresponding to the signal-to-noise ratio $d_{o}$ of the loudest trigger obtained in the search, i.e., $\mathcal{F}_{o}=2+\frac{1}{2} d_{o}^{2}$. Then, using formula (10) for detection probability, we calculate the signalto-noise ratio $d_{\mathrm{ul}}$ of the gravitational-wave signal so that there is probability $\mathcal{P}$ that it crosses threshold $\mathcal{F}_{o} ; d_{\mathrm{ul}}$ is the desired $\mathcal{P}$ confidence upper limit. For several independent searches, the relation between the confidence $\mathcal{P}$ and upper limit $d_{\mathrm{ul}}$ is given by 
Table 2. Maximal signal-to-noise ratios of events at various stages of the data analysis procedure. The maxima are quoted for each stage of the search separately and in general they correspond to different triggers. 'Linear parametrization' means search using linear parametrization introduced in section 2.3, 'astrophysical parametrization' means verification using astrophysical parameters and precise ephemeris of the detector, 'different data' denotes verification in a different set of data, 'longer data' means verification in four-day stretch of data, 'all data' means verification in the whole 13-day set of data.

\begin{tabular}{llllll}
\hline \multicolumn{5}{c}{ Maximal signal-to-noise ratios } \\
\hline \multirow{2}{*}{ Search } & $\begin{array}{l}\text { Linear } \\
\text { parametrization }\end{array}$ & $\begin{array}{l}\text { Astrophysical } \\
\text { parametrization }\end{array}$ & $\begin{array}{l}\text { Different } \\
\text { data }\end{array}$ & $\begin{array}{l}\text { Longer } \\
\text { data }\end{array}$ & $\begin{array}{l}\text { All } \\
\text { data }\end{array}$ \\
\hline I & 8.01 & 8.19 & 7.68 & 8.02 & 7.35 \\
II & 8.28 & 8.29 & 8.05 & 7.91 & 7.48 \\
III & 8.48 & 8.45 & 7.38 & 8.90 & 7.70 \\
\hline
\end{tabular}

$$
\mathcal{P}=1-\prod_{i=1}^{L}\left(1-P_{D}\left(d_{\mathrm{ul}}, \mathcal{F}_{o i}\right)\right),
$$

where $\mathcal{F}_{o i}$ is the threshold corresponding to the loudest event in the $i$ th search and $L$ is the number of searches. Here, $\mathcal{P}$ is the probability that a signal of signal-to-noise ratio $d_{\mathrm{ul}}$ crosses the threshold $\mathcal{F}_{o i}$ for at least one of the $L$ independent searches.

In order to translate our upper limit for the signal-to-noise ratio to upper limit $h_{\mathrm{ul}}$ for gravitational-wave amplitude, we assume that our source of a continuous gravitational wave is a spinning neutron star modelled as a triaxial ellipsoid. Since we have searched the whole sky we cannot assume a particular position of the source and a specific polarization of the gravitational wave. Therefore, to relate amplitude of the wave and the signal-to-noise ratio we used the averaged expression given by equation (93) of [3]. Moreover, as we do not know the frequency of the gravitational-wave signal for spectral density we take its average $\bar{S}_{o}$ over the bandwidth. Thus $h_{\mathrm{ul}}$ and $d_{\mathrm{ul}}$ are related by the following formula:

$$
h_{\mathrm{ul}}=\frac{5}{2} \sqrt{\frac{\bar{S}_{o}}{T_{o}}} d_{\mathrm{ul}} .
$$

Assuming confidence of $90 \%$ and taking the signal-to-noise ratios of the loudest events for astrophysical parametrization equal to 8.19, 8.29 and 8.45 for searches I, II and III, respectively, we find that for the first search $d_{\mathrm{ul}}=9.5$ whereas for all three searches $d_{\mathrm{ul}}=8.5$. These correspond to average dimensionless amplitudes of $1.0 \times 10^{-22}$ and $9.4 \times 10^{-23}$, respectively. Since in the first search the data conformed very well to a Gaussian distribution we consider the upper limit for the first search as the most reliable.

Consequently, we have the following conclusion from our search: in the frequency band from $921.00 \mathrm{~Hz}$ to $921.76 \mathrm{~Hz}$, for spin-down range from $-2.36 \times 10^{-8} \mathrm{~Hz} \mathrm{~s}^{-1}$ to $+2.36 \times$ $10^{-8} \mathrm{~Hz} \mathrm{~s}^{-1}$ and for signals coming from any sky direction and of any polarization, the dimensionless amplitude of the gravitational wave from a continuous source is less than $1 \times 10^{-22}$ with $90 \%$ confidence.

This upper limit is higher than the one we previously reported [7]. The reason is that in [7] to evaluate the upper limit on amplitude we used maximal gravitational-wave amplitude and the minimal spectral density of the detector noise instead of the average quantities that we use here. In [11], a more sophisticated method to determine the upper limit was developed. In [11], instead of assuming Gaussian distribution of the data the probability of detection was obtained by injecting signals to the data. 


\section{Acknowledgments}

The work of P Jaranowski, A Królak, K M Borkowski and M Pietka was supported in part by the KBN (Polish State Committee for Scientific Research) grant no 1 P03B 029 27. We would also like to thank the Interdisciplinary Center for Mathematical and Computational Modelling of Warsaw University for computing time.

\section{References}

[1] Astone P et al 1993 Phys. Rev. D 47362

[2] Astone P et al 2002 Phys. Rev. D 65022001

[3] Jaranowski P, Królak A and Schutz B F 1998 Phys. Rev. D 58063001

[4] Jaranowski P and Królak A 1999 Phys. Rev. D 59063003

[5] Jaranowski P and Królak A 2000 Phys. Rev. D 61062001

[6] Astone P, Borkowski K M, Jaranowski P and Królak A 2002 Phys. Rev. D 65042003

[7] Astone P et al 2003 Class. Quantum Grav. 20 S665-76

[8] Tretter S A 1976 Introduction to Discrete Time Signal Processing (New York: Wiley)

[9] http://www.astro.uni.torun.pl/kb/AllSky/AllSky.html

[10] Lagarias J C, Reeds J A, Wright M H and Wright P E 1998 SIAM J. Optim. 9112

[11] Abbott B et al (LIGO Scientific Collaboration) 2004 Phys. Rev. D 69082004 